\title{
Cloud-top entrainment instability?
}

\section{B. STEVENS}

Max-Planck-Institut für Meteorologie, Bundesstrasse 53, 20146 Hamburg, Germany

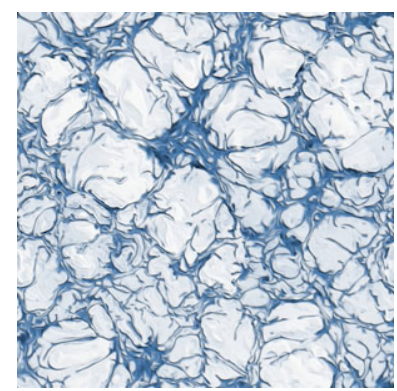

Mixing processes at cloud boundaries are thought to play a critical role in determining cloud lifetime, spatial extent and cloud microphysical structure. High-fidelity direct numerical simulations by Mellado (J. Fluid Mech., 2010, this issue, vol. 660, pp. 5-36) show, for the first time, the character and potency of a curious instability that may arise as a result of molecular mixing processes at cloud boundaries, an instability which until now has been thought by many to control the distribution of climatologically important cloud regimes.

Key words: buoyancy-driven instability, moist convection, turbulent mixing

\section{Introduction}

The planetary energy balance, and hence Earth's climate, in large part depends on the infrared emissivity and the shortwave reflectivity of the atmosphere. Both, but especially the latter, are strongly influenced by clouds. On average, our planet is twice as reflective as it would be were it not for the direct presence of clouds. Hence, it is not surprising that uncertainties in how clouds may change underlie most of the uncertainty in climate projections. Of the varied cloud types, shallow marine boundary-layer clouds have an especially large impact on both the present energy balance, and predictions of how it might change (Bony \& Dufresne 2006).

Peculiar among these clouds is a regime transition between nearly solid cloud decks, preferentially found over the regions of cold water associated with the eastern upwelling currents of the subtropical ocean gyres, and scattered cumuliform clouds that prevail over warmer waters and in the trade winds. This regime transition challenges our understanding of factors that control cloudiness, and hence climate. One long-standing idea is that this transition is selected as a result of a cloud-top entrainment instability (CTEI) thought to develop when the criterion for buoyancy reversal is satisfied. As the physics underlying such a criterion depends on molecular mixing processes between two-phase, two-component, fluids at very high Reynolds number, it has proved difficulty to address at a fundamental level. As a result the idea of CTEI, introduced more than 30 years ago (Randall 1980; Deardorff 1980) has remained controversial.

\section{Overview}

For a 'buoyancy reversing' fluid the sign of the density change, per differential amount of mixing, depends on the saturation state of the mixed fluid, as illustrated 


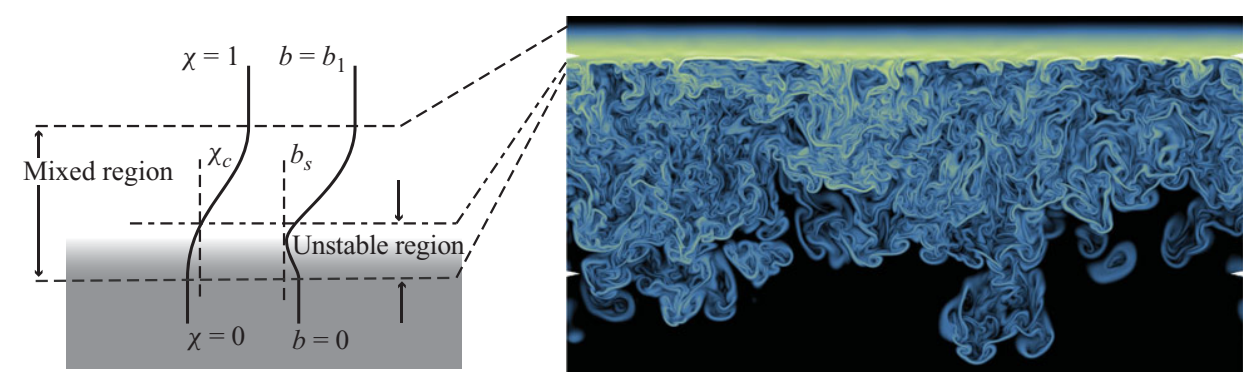

FIGURE 1. Evaporative-driven layer. Schematic on the left shows the buoyancy versus the mixing fraction within the enlarged mixing region. The range of negatively buoyant mixing fraction, defining the unstable sublayer, is given by $\chi \in\left[0, \chi_{c}\right]: b_{s}$ defines the most negatively buoyant mixture. Evolution within a vertical plane of the logarithm of the scalar dissipation rate of the developing turbulent layer (from figure 3 of Mellado (2010)).

by the schematic in figure 1 . As one moves upwards the mixing fraction $\chi$ increases, but initially the fluid remains saturated and the buoyancy decreases. At some point, mixtures become just saturated. Thereafter further increases in $\chi$ are not accompanied by further evaporation and buoyancy increases with $\chi$, eventually becoming positive again. The mixing fraction $\chi_{c}$ separates negatively from positively buoyant mixtures and defines a mixing sublayer that is convectively unstable. This capacity for buoyancy reversal is a fundamental characteristic of the atmosphere (Stevens 2005).

The exact criterion for the onset of buoyancy reversal has evolved in its details over the last half century, and roughly correlates with the climatological transition between the regions of solid stratiform clouds and the regions of scattered cumuliform clouds. This point, and the tendency of large-eddy simulation (LES) to produce a rapid desiccation of the cloud layer when the buoyancy reversal criterion is met, was demonstrated by Randall (1980) and Deardorff (1980) respectively. Since these seminal studies the idea that the stratiform cloud layer becomes unstable under conditions of buoyancy reversal has dominated thinking on the subject of marine boundary-layer cloud transitions.

The idea has nonetheless remained controversial. Researchers were quick to point out that (i) the stratiform clouds are frequently found in regions whose large-scale thermodynamic conditions appear to support buoyancy reversal and hence the main criterion for CTEI (Kuo \& Schubert 1988); (ii) the measured rates of mixing at the top of stratiform cloud layers also show little evidence of accelerated mixing in conditions of buoyancy reversal (Stevens et al. 2003); (iii) if care is devoted to the representation of cloud-top mixing process, large-eddy simulations are able to maintain stratiform clouds under buoyancy reversal conditions; and (iv) the argument that there is a buoyancy reversal instability, has remained largely heuristic - formal derivation of the criterion as a linear instability has until recently (Mellado et al. 2009), been absent. These counter lines of argument, combined with a lack of detailed information about the mixing processes that may underlie any potential instability, have left the field deadlocked on the subject. Hence the key question, as to whether microscopic mixing processes can decide the macroscopic fate of fields of clouds has remained open, at least until now.

In a series of three papers, which begin with the linear stability analysis of a threelayer fluid, and culminating in the thorough analysis of three-dimensional simulations 
presented in this volume, Dr. Mellado and his colleagues convincingly demonstrate that while buoyancy reversing layers are formally unstable, the instability likely plays a minor role in the evolution of real clouds. Mellado (2010) analyses three simulations characterised by different degrees of buoyancy reversal. The use of high-order methods and a large computational mesh (approaching $10^{10}$ points) allows the simulations to represent relatively high-Reynolds-number flows (Taylor-scale Reynolds numbers of about 200, equivalently Rayleigh numbers approaching $10^{9}$ ) with great fidelity (see the right panel of figure 1). Convective plumes accelerate away from the cloud-top mixing region into the underlying saturated fluid.

In the simulations the cloud-top mixing interface can still be identified by a thin layer, defined by the region of transition from the irrotational unsaturated upper fluid to the saturation interface topping the lower fluid. Within this layer, scalar gradients are large and molecular transport processes dominate. The analysis by Mellado (2010) suggests that the thickness of the layer reflects a balance between its rate of thickening as a result of molecular processes and the rate at which unstable fluid elements are produced and convect away from the layer. The development of turbulence in the lower layer enhances the transport of mixed fluid away from the interface, and prevents the diffusive layer from thickening. This results in a constant layer thickness, $h$. Because the diffusivity, $\kappa$ is constant, the layer deepens with a fixed velocity, $w_{e} \simeq \kappa / h$, which defines the entrainment rate. Although this rate of deepening is faster than what one would have without turbulence, the increase of turbulence intensity in the lower fluid (associated with the growth in the length scale of the convecting plumes) does not feedback on the interfacial dynamics so as to further enhance mixing, for instance by further thinning the interface.

The idea of cloud-top entrainment instability hinges on this large-scale feedback, wherein the turbulence developing as a result of mixing accelerates the mixing, which increases the turbulence intensity and so on, leading to the rapid desiccation of the layer. Through the development of new and fundamental insights into the nature of mixing processes at cloud boundaries and, in particular, the interplay of molecular and turbulent processes, the analysis of Mellado (2010) suggests that buoyancy reversal is not a sufficient condition for the rapid demise of stratiform cloud layers.

To be sure, simplifications are still necessary to simulate flow regimes relevant to the atmosphere. For instance, the simulations performed and analysed by Mellado (2010) focus only on fluid dynamical processes that develop in the interfacial region separating two fluids whose mixtures are buoyancy reversing. The extent of the domain, of order $10 \mathrm{~m}$, is much smaller than the outer scale of real cloud layers, which is typically 100-fold larger. However, over the range of Reynolds numbers accessible to computations the convective layer is shown to develop self-similarly, thereby encouraging the simple rescaling of the problem to atmospherically relevant regimes. And while casting the question as a two-fluid problem permits the use of a mixing-fraction formulation (Bretherton 1987; Mellado et al. 2010), it also requires the scalar diffusivity of temperature, moisture and condensate to be identical and droplets to remain in perfect suspension within the fluid. Thus the effects of differential diffusion and droplet sedimentation are neglected. However, none of these simplifications appear to be important to the basic conclusions of this work, which is that for conditions encountered in the atmosphere the diffusivity limits the rate of mixing and hence paces the development of the developing convective layer within the cloud. 


\section{Future}

Many of these simplifications define important avenues for future investigation. For instance, when does turbulence become important in determining the thickness of the inversion layer? To explore this question, one would like to perform simulations wherein the turbulence intensity within the cloud layer can be forced by means other than buoyancy reversal. Such situations are common in the atmosphere, where turbulence arises from radiative cooling at cloud top, surface heating and large-scale shearing motions in the horizontal wind. Understanding these cases will be important to understanding more generally what controls entrainment rates, and hence cloudiness in real cloud layers. Another question involves the interplay of droplet dynamics with the turbulence field. What is the impact of droplet sedimentation, and inertial effects? The rate at which liquid water is made available to mix with the unsaturated air above is critical to the evolution of the entrainment region, which raises the question as to the degree such processes regulate the mixing in this region.

A further challenge is to incorporate these results into the LES representations of the cloud-topped atmospheric boundary layer. To the extent the results of Mellado (2010) hold more generally, it suggests that LES as traditionally formulated is fundamentally incapable of representing processes that depend on buoyancy reversals at cloud boundaries. To address such questions with LES will thus require the development of new methods to both track the mixing interface and model the mixing there, in part based on insights developed through studies such as Mellado (2010).

\section{References}

Bony, S. \& Dufresne, J.-L. 2006 Marine boundary layer clouds at the heart of tropical cloud feedback uncertainties in climate models. Geophys. Res. Lett. 32, L20806.

BRETHERTON, C. S. 1987 A theory for nonprecipitating moist convection between two parallel plates. Part I. Thermodynamics and linear solutions. J. Atmos. Sci. 44, 1809-1827.

Deardorff, J. W. 1980 Cloud top entrainment instability. J. Atmos. Sci. 37, 131-147.

Kuo, H.-C. \& Schubert, W. H. 1988 Stability of cloud-topped boundary layers. Q. J. R. Meteor. Soc. 114, 887-916.

Mellado, J. P. 2010 The evaporatively-driven cloud-top mixing layer. J. Fluid. Mech. 660, 5-36.

Mellado, J. P., Stevens, B., Schmidt, H. \& Peters, N. 2009 Buoyancy reversal in cloud-top mixing layers. Q. J. R. Meteor. Soc. 135, 963-978.

Mellado, J. P., Stevens, B., Schmidt, H. \& Peters, N. 2010 Two-fluid formulation of the cloud-top mixing layer for direct numerical simulation. Theor. Comput. Fluid Dyn. doi:10.1007/s00162010-0182-X.

Randall, D. A. 1980 Conditional instability of the first kind upside-down. J. Atmos. Sci. 37, $125-130$.

Stevens, B. 2005 Atmospheric moist convection. Annu. Rev. Earth Planet. Sci. 33, 605-643.

Stevens, B., Lenschow, D. H., Ian Faloona, C.-H. M., Lilly, D. K., Blomouist, B., Vali, G., Bandy, A., Campos, T., Gerber, H., Haimov, S., Morley, B. \& Thorton, D. C. 2003 On entrainment in nocturnal marine stratocumulus. Q. J. R. Meteor. Soc. 129, 3469-3492. 